\title{
Targeting myostatin could prevent bone destruction in inflammatory arthritis
}

Myostatin has been indirectly linked with bone turnover through its influence on muscle, but new evidence highlights a direct role for this transforming growth factor- $\beta$ family member in inflammatory joint destruction, as occurs in rheumatoid arthritis (RA). The report in Nature Medicine also indicates that deficiency or inhibition of myostatin strongly reduces this damage.

"The effects of myostatin on bone are not merely an indirect mechanical effect, as previously thought, but a direct effect on osteoclasts and therefore on bone resorption," explains corresponding author Thomas Pap. "The findings raise the question of whether it makes sense to inhibit myostatin therapeutically in RA."

Pap and colleagues systematically investigated the expression of myostatin in vivo, its regulation by inflammatory mediators in vitro, and the effects of the absence of myostatin in two different models of RA. They found that myostatin is expressed at high levels in synovial tissue from patients with RA and from TNF transgenic (hTNFtg) mice, which develop destructive inflammatory arthritis. Moreover, expression of myostatin in human synovial cells increased upon exposure to proinflammatory cytokines. In vitro studies demonstrated that myostatin is a direct paracrine and autocrine regulator of receptor activator of nuclear factor- $\kappa \mathrm{B}$ ligand (RANKL)-induced osteoclast development. "One of our main findings was that myostatin exerts its effects on osteoclastogenesis through the SMAD pathway," reports Pap.

Turning to mouse models of RA, the investigators found that myostatin deficiency $\left(M s n^{--}\right)$or treatment with myostatin-specific antibody ameliorated disease severity, particularly osteoclast formation and bone erosion, in hTNFtg mice. Joint destruction was similarly reduced in $\mathrm{Mstn}^{-/-}$mice with serum transfer-induced arthritis compared with wild-type mice.

Together, the results highlight the potential of myostatin-directed pharmacological treatment for RA. "Therapeutically inhibiting myostatin might not only increase muscle mass but also inhibit bone destruction," concludes Pap.

Sarah Onuora

\section{Original article}

Dankbar, B. et al. Myostatin

is a direct regulator of osteoclast

differentiation and its inhibition reduces inflammatory joint

destruction in mice. Nat. Med. doi:10.1038/nm.3917 